\section{"Normal" Leucocytosis}

The leucocyte count in the peripheral venous blood of most healthy adults under sedentary conditions usually lies between 4,000 and $10,000 / \mu 1$. Small deviations from these limits $( \pm 1,000)$ may be encountered, but it is rare to find that the leucocyte count of healthy adults is much outside this range. There tends to be a diurnal variation in the leucocyte count, it being slightly higher in the afternoon than in the early morning. ${ }^{1}$ Strenuous exercise can raise the count temporarily, as can an injection of adrenaline. This is due to mobilization of leucocytes from the large marginal pool (distributed along the walls of blood vessels) into the circulating pool, ${ }^{2} 3$ the latter being sampled when peripheral venous blood is taken for counting. It has also been reported that emotional tension may be associated with a raised leucocyte count ${ }^{4}$ and that cigarette smokers, especially if they inhale, tend to have higher leucocyte counts than nonsmokers. 5

A recent report by H. N. Ward and E. H. Reinhard ${ }^{6}$ emphasizes the extent to which the leucocyte count may be raised well above the generally accepted upper limit of $10,000-11,000 / \mu 1$. in some apparently physically healthy people. They record the findings in a group of 35 Caucasian patients (both men and women, aged 22-65 years) whose leucocyte count had been persistently above $11,000 / \mu l$. for an average period of 35 months before referral for haematological opinion. These patients either had no demonstrable physical disease or their diseases were considered insufficient to explain the leucocytosis. The presenting symptoms were largely functional. Most of the counts were between 10,000 and $20,000 / \mu 1$. (average about 15,000), and the increase was due entirely to an increase in the neutrophils, which were above the generally accepted normal upper limit of $7,500 / \mu 1$. Ten patients had a slight left shift, with band forms and occasional metamyelocytes and myelocytes, but qualitative abnormalities such as toxic granulation and Döhle bodies were not seen. The remainder of the blood picture was normal except for a slightly raised platelet count in two patients.

After referral these patients were followed up for an average period of about seven years ( 7 months to 19 years). One patient was lost to follow-up, and, of the remaining 34, 11 had not developed any significant illness during the follow-up period. Disease became evident in four patients. One developed Hodgkin's disease nine years after the first finding of a leucocytosis. The second developed a fever of unknown origin after about eight years and on laparotomy showed multiple granulomas in the liver from which tubercle bacilli were cultured. The third died after 18 months from what appeared to be vasculitis associated with cryoglobulinaemia. And the fourth patient developed rheumatoid arthritis. In none of the four patients was it clear that these diseases were related to the raised leucocyte counts found initially. The illnesses which appeared in the remaining patients would not have accounted for a persistent neutrophilia. Aspirates of bone marrow were obtained from 24 patients: 15 were thought to be normal, 8 showed slight to moderate granulocytic hyperplasia or a left shift in the granulocytic series, and 3 showed a slight eosinophilia. The neutrophil alkaline phosphatase test was normal in most of the 19 patients on whom it was carried out.

A persistent and unexplained neutrophilic leucocytosis raises the possibility of an early stage of chronic myeloid (granulocytic) leukaemia, but in none of Ward and Reinhard's patients did this disease become evident. They therefore emphasize that these patients with unexplained neutrophilic leucocytosis were not in an early stage of chronic granulocytic leukaemia. Two of the patients had been severely frightened by being told originally that they had a disorder "bordering on leukaemia." One patient is of particular interest in that his leucocyte count had been as high as $43,200 / \mu 1$., and over a period of seven years, during which blood counts were done every four months, his leucocyte count was usually above $25,000 / \mu 1$. This patient is said to have remained in perfect physical health except for continued anxiety about his "high white blood cell count," which it had not been possible to allay by constant reassurance. It is of interest that 25 patients in the series were known to smoke cigarettes. Of these only one had stopped smoking, and his leucocyte count had returned to normal. An accelerated granulocytic turnover rate has been found ${ }^{7}$ in some patients with idiopathic leucocytosis, suggesting that there is an increased input of neutrophils into the blood as well as an increased outflow.

Investigating patients who are found in a laboratory test to have an unexplained deviation from the generally accepted normal range carries with it the risk of provoking needless anxiety and should be carried out, if at all, with great circumspection. The significance of an "abnormal" test in a person who is apparently well clinically should be related to the findings as a whole and ultimately must be based on careful clinical judgement. The so-called normal values embrace a range covering about $95 \%$ of the population. Thus up to $5 \%$ of people may deviate from the normal range without being demonstrably ill.

1 Jeffrey, H. C., Fournal of the Royal Army Medical Corps, 1961, 107, 93. 2 Athens, J. W., et al., Fournal of Clinical Investigation, 1961, 40, 989.

3 Athens, J. W. et al, Fournal of Clinical Investigation, 1961, 40, 159

4 Milhorat, A. T., Small, S. M., and Diethelm, O., Archives of Neurology and Psychiatry, 1942, 47, 779.

${ }^{5}$ Corre, F., Lellouch, J., and Schwartz, D., Lancet, 1971, 2, 632.

Ward, H. N., and Reinhard, E. H., Annals of Internal Medicine, 1971, $75,193$.

7 Athens, J. W., Haab, O. P., and Raab, S. O., fournal of Clinical Investigation, $1965,44,778$.

\section{Paroxysmal Tachycardia}

Our understanding of cardiac dysrhythmias and conduction defects has been greatly increased in the last few years by the study of intracardiac electrograms. ${ }^{1-3}$ Direct recordings from the bundle of His in the intact human heart were first obtained in 1960 by G. Giraud and colleagues ${ }^{4}$ and subsequently by $\mathrm{H}$. Watson and his colleagues in Dundee. 5 Since then it has been shown that the timing of depolarization of the bundle of His in relation to that of the right atrium and right ventricle can be recorded safely and easily during cardiac catheterization. The site of delays in atrioventricular conduction and the presence of reversed conduction can be defined by this technique.

A multipolar electrode catheter is used to record the initiation of depolarization of the bundle of His within the PR interval of the simultaneously recorded surface electrocardiogram. By this means atrioventricular conduction can be divided into two segments. The first interval, P-H (normal 80 to 140 milliseconds), is a measure of intra-atrial and atrioventricular nodal conduction time. The second interval, H-Q (normal 35 to $55 \mathrm{msec}$ ), reflects transmission within the ventricular conducting tissue. These events all 